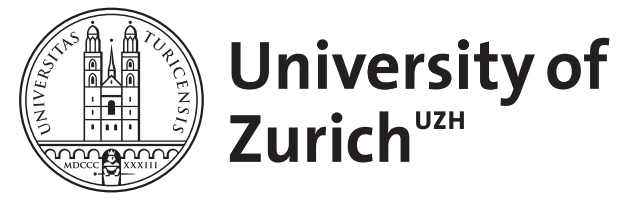

Zurich Open Repository and Archive

University of Zurich

University Library

Strickhofstrasse 39

CH-8057 Zurich

www.zora.uzh.ch

Year: 2003

\title{
Theorien der Internationalen Beziehungen
}

Marti, Urs

Posted at the Zurich Open Repository and Archive, University of Zurich

ZORA URL: https://doi.org/10.5167/uzh-54557

Book Section

Published Version

Originally published at:

Marti, Urs (2003). Theorien der Internationalen Beziehungen. In: Kohler, G; Marti, U. Konturen der neuen Welt(un)ordnung. Beiträge zu einer Theorie der normativen Prinzipien internationaler Politik. Berlin/New York: de Gruyter, 62-79. 


\section{Theorien der internationalen Politik}

URS MARTI

Die ökonomischen Globalisierungsprozesse der Gegenwarr wirken sich auf Gesellschaft, Recht und Politik in einer Weise aus, die häufig als Krise erlebt wird. Können Theorien internationaler Beziehungen zux Bewältigung solcher Krisen beitragen?' Oder sind ihre widersprüchlichen Antworten auf Fragen nach politischen Gestaltungsmöglichkeiten in einer sich verändernden Welt, in der viele Grenzen neu gezogen werden, selbst Ausdruck einer Krise? Lässt die Vielfalt der von thnen angebotenen Erklärungsmodelle auf die fehlende wissenschaftliche Reife der Disziplin schließen, oder ist sie ein Indiz für die politische Instrumentan lisierung von Theorien? Die Wissenschaft der internationalen Beziehungen biete der politischen Praxis Orientierung und Legitimation in einer sich verändernden Welt (Meyers, 1990, S. 48); dass sich darin auch politische Motive artikulieren, versteht sich von selbst. Zwischen realistischen Theorien und konservativen politischen Richtungen bestehen offensichtlich Affinitäten, ebenso zwischen Institutionalismus und Liberalismus oder zwischen Strukturalismus und Radikalismus. Unterschiedliche theoretische Ansätze lassen sich jedoch mit gegensätzlichen polim tischen Präferenzen nicht hinreichend erklären.

Zu den wichtigsten Unterscheidungsmerkmalen gehört die Bestimmung der Akteure internationaler Polirik, ihrer Motive und Handlungsmöglichkeiten sowie des Milieus, worin sie agieren. Ebenso gehören dazu Annahmen über die menaschliche Natur, über die Funktionsweise des internationalen Systems sowie über die Regeln von Konflikt, Kooperation und Abhängigkeit. Bis in die 70er Jahre des 20. Jahrhunderts war die Theorie der Internationalen Beziehungen dominiert von Realismus und Liberalismus, seither wurden marxistisch orientierte Ansätze als drit. tes Paradigma vermehrt zur Kenntnis genommen. In zahlreichen Lehrbüchern werden drei Hauptrichtungen unterschieden, wobei die erste Schule als Realismus bezeichnet wird, die zweite als Liberalismus, Institutionalismus, Pluralismus, Interdependenz- oder Weltgesellschafts-Theorie, die dritte als Strukturalismus, Radikalismus, Globalismus, Dependenz-oder Weltsystem-Theorie.2

Im realistischen Modell sind Staaten die hauptsächlichen Akteure; sie werden als homogene Gebilde sowie als rationale Nutzenmaximierer verstanden, deren Grundmotiv die Sorge um die nationale Sicherheit oder das nationale Interesse

1 Es ist "möglicherweise sinnvoll, in wissenschaftsgeschichtlicher Finsicht die Lehre von den Internationalen Beziehungen stärker als [...] , Kriseninterpretations" und Xrisenbewältigungswissenschaft' zu begreifen", so urteilt Meyers, 1990, S. 61

2 Vgl. Banks, 1985; Viotti/Kauppi, 1990, S. 5-11; Meyers, 1990, S. 57-63; Holsti, 1995, S. 42-47; Smith, 1995, S. 18-21; Waever, 1996; Baylis/Smith, 1997, S. 3-5, 109-163; Lemke, 2000, S. 5-54. darstellt. Weltpolitik wird in der Perspektive des Realismus vorwiegend von militärischen Kräfteverhältnissen gestaltet; das internationale System präsentiert sich
als strukturelle Anarchie.

Liberale Theorien befassen sich mit staatlichen und nicht-staatlichen Alkteuren wie internationalen Organisationen oder multinationalen Unternehmen. Staaten sind in dieser Sichtweise nicht homogen, sondern setzen sich zusammen aus konkurrierenden Gruppen; staatliche Politik resultiert aus Kompromissen und nicht aus rationaler Entscheidung; internationale Politik ist nicht ausschließlich eine militärische, sondern auch eine ökonomische, soziale und ökologische Angelegenheit; das internationale System wird als eine durch komplexe Interdependenzen konstitutierte Weligesellschaft verstanden.

Für den Globalismus kommen als Akteure Klassen, Staaten, Gesellschaften und nicht-staatliche Organisationen in Betracht, sie alle agieren nicht völlig autonom, sondern als Teile des kapitalistischen Weltsystems, welches nur unter Finbezug ner Geschichte analysiert werden kann. Politik wird als Resultat vielfältiger Abhängigkeiten in einem System ungleicher Entwicklung von Gesellschaften thematisiert, wobei die entscheidenden Faktoren der Weltpolitik ökonomischer Arr sind.

Ob sämtliche Erklärungsmodelle den drei Großtheorien zugeordnet werden können, ist zwar umstritten, doch wird eine gewisse Komplexitätsreduktion al sinnvoll beurteilt; wobei feinere Klassifikationen sich von Fall zu Fall als hilfreich erweisen. Eine detailliertere Einteilung ergibt sich, wenn Weltmodelle zugrunde gelegt werden. Innerhalb des Realismus basieren det traditionelle Realismus und der Neorealismus auf dem Modell einer anarchischen Statenwelt, der liberale Realismus oder Rationalismus der "Englischen Schule" auf dem Modell einer rechtlich verfassten Staatenwelt. Im Liberalismus lassen sich drei Richtungen unterscheiden. Der Institutionalismus geht vom Modell transnationaler Interdependenz und Kooperation aus. Der Internationalismus bält sich an das Modell einer globalen Friedens- und Rechtsordnung. In einer radikaleren Variante wird das Schwergewicht auf die inner- und überstaatliche Demokratisierung gelegt. In Folgenden halte ich mich an diese Einteilung und verweise in einem weiteren Abschnitt auf neuere, so genannt reflexive Ansätze.

\section{Realismus und Neorealismus}

Der Realismus beruft sich auf eine über zweitausendjährige Tradition. In Thukydides' Geschichte des Peloponnesischen Kriegs (V/89) verkünden die Athener den Meliern, Recht könne nur $z$ wischen Gleichmächtigen bestehen. Als neuzeitliche Vertreter des Realismus werden Machiavelli und Hobbes genannt, zu erinnern ist auch an Rousseau, der 1761 das vom Abbé de Saint-Pierre 1713 veröffentlichte Projekt einer europäischen Friedensordnung kritisiert (Rousseau, 1964, S. 563 600). Im 20. Jahrhundert konnte die realistische Schule Einfluss gewinnen, nachdem der liberale Idealismus angesichts des Scheiterns des Völkerbunds, der Heraufkunft des Nationalsozialismus und des Ausbruchs des zweiren Weltkriegs seine Überzeugungskraft verloren hatte. Die Kritik idealistischer Friedenshoffnungen 
verweist auf die Tatsache, dass auf internationaler Ebene jener Leviathan fehlt, der gemäß der Lehre von Hobbes den kriegerischen Naturzustand auf nationaler Ebene zu beenden vermag. Der Staat bleibt daher der Hauptakteur internationaler Politik. Als legitimer Vertreter eines kollektiven Willens hat er das Überleben des von ihm repräsentierten Kollektivs zu sichern und ist dabei an keine übergeordneten Normen gebunden.

Hans Morgenthau versteht die Geschichte des neuzeitlichen politischen Denkens als Auseinandersetzung $z$ wischen einer der Aufflärung verpflichteten Schule und einer Schule, die vom menschlichen Wesen, "wie es wirklich ist", sowie von historischen Prozessen, „wie sie den Tatsachen entsprechen ${ }^{\text {" }}$, ausgeht. Politik wird ihm zufolge von objektiven, in der unveränderlichen menschlichen Natur wurzelnden Gesetzen beherrscht. Machtinteressen determinieren die internationale Politik, wobei die Formen der Macht in der Geschichte variieren. Möglicherweise wird sich das Interesse dereinst vom Nationalstaat lösen und übernationale Einheiten werden entstehen. Zwischen moralischen Prinzipien und den Anforderungen an erfolgreiches politisches Handeln besteht jedoch ein unaufhebbarer Gegensatz; abstrakte moralische Regeln können auf staatliches Handeln, das allein dem Gebot nationaler Selbsterhaltung gehorcht, nicht angewandt werden. Nationen mögen versucht sein, ihre Politik unter Berufung auf universelle moralische Ziele zu rechtfertigen, aber diese Rechtfertigung ist immer nur Vorwand. Die Fixierung auf den Aspekt der Macht erlaubt es schließlich, einen eigenständigen Bereich der Politik von Ökonomie, Jurisprudenz und Moral abzutrennen (Morgenthau, 1963, S. 48-57).

Zu den theoretischen Schwächen des traditionellen Realismus gehört indes gerade die Geringschätzung ökonomischer Faktoren. Die zunehmende zwischenstaatliche Tnterdependenz und de stêgende Bedeutung transnationaler ökonomischer Akteure stellen für eine Theorie, die den von Morgenthau formulierten Grundsätzen verpflichtet ist, eine Herausforderung dar. In den 70er Jahren entsteht mit dem Neorealismus oder strukturalen Realismus eine neue Schule. Der ältere Realismus ist laut Kenneth Waltz auch an wissenschaftstheoretischen Problemen gescheitert. Nur hypothetisch kann nämlich von einer Autonomie des politischen Bereichs gesprochen werden, sie folgt aus der Konstruktion eines internationalen Systems (W/altz, 2000b, S. 1525-1528). Anders als der traditionelle Realismus, der die Gesetze der Politik aus der menschlichen Natur herleitet, verweist Waltz auf die systemischen Zwänge der internationalen Ordnung. Diese Zwänge, wie sie von der anarchischen Struktur der internationalen Politik ausgehen, bringen die Staaten trotz unterschiedlicher Verfassungen und Zielsetzungen dazu, sich in der Außenpolitik gleich zu verhalten und zum $Z$ weck ihrer eigenen Sicherheit militärische. Macht zu akkumulieren. In dieser Sichtweise.ist das. System letztlich allmächtig, die Staaten können sich zwar mit mehr oder weniger Macht am Kampf beteiligen, sie besitzen aber nicht die.Macht die strukturellen Bedingungen zu verändern (Waltz, 1979; Burchill 1996b). Obgleich der Neorealismus seit den 80er Jahren auf wachsende Kritik stößt (Keohane, 1986), ist sein Einfluss beträchtlich geblieben, wie die Annäherung des institutionalistischen Neoliberalismus an den Neorealismus zeigt (Baldwin, 1993; Kegley, 1995). Generell wird dem
Realismus eine selektive Wahrnehmung der Realität vorgeworfen, doch werden bestimmte Einsichten in die Mechanismen von Anarchie und Machtkampf auch von Kritikern geteilt (Donnelly, 2000, S. 193-200).

\section{Rationalismus}

Realistische wie kosmopolitische Theorien geben von der Dynamik internationaler Beziehungen ein falsches Bild. Diese Auffassung wird seit den 50er Jahren von Theoretikern vertreten, die für einen rationalistischen oder internationalistischen Mittelweg plädieren (Linklater, 1996a; Dunne, 1998). Hedley Bull, der 1977 „The Anarchical Society" publiziert, versteht die zwischenstaatlichen Verhältnisse gleichsam als regulierte Anarchie, als Gesellschaft, für deren Mitglieder bestimmte Regeln verbindlich sind. Das Modell des zwischenstaatlichen Naturzustands ist ihm zufolge dann brauchbar, wenn es nicht im Sinn von Hobbes, sondern von Locke vestanden wird (Bull, 2000, S. 608-611). In einem lockeschen Naturzustand können Menschen überleben; in einer globalen anarchischen Gesellschaft sind Staaten fähig, zu kooperieren und allgemein gültige Normen auch dann zu befolgen, wenn kein Weltstaat sie dazu zwingt. Letztlich erweist sich aber die Analogie zwischen Menschen und Staaten als problematisch; die Bedingungen, unter denen Staaten Ordnung anstreben und verwirklichen, sind mit der Situation der Individuen nicht vergleichbar.

Eine internationale Gesellschaft existiert, sobald mehrere Staaten im Bewrusstsein gemeinsamer Interessen und Werte in ihren gegenseitigen Beziehungen eine Reihe von Regeln respektieren und sich am Aufbau gemeinsamer Institutionen beteiligen. Die internationale Gesellschaft kann $z$ war auf die internationale Politik bis zu einem gewissen Grad ordnend einwirken, aber die durch sie gestüzzte Ordnung ist prekär. Die Idee der Weltordnung hat für Bull'thormativen Vorrang vor jener der internationalen Ordnung, er begreift die Welt als eine aus Individuen gebildete "great society of all mankind". Es ist denn auch nicht zuletzt sein Sensorium für Fragen globaler ökonomischer und sozialer Gerechtigkeit, das ihn die Schwächen realistischer wie kosmopolitischer Modelle erkennen lässt. Ordnung ohne Gerechtigkeit bleibt unstabil; eine stabile Weltordnung kann aus der Gesellschaft der Staaten nur dann entstehen, wenn zuvor eine radikale Umverteilung von Wohlstand, Ressourcen und Macht zwischen reichen und armen Staaten stattfindet, da andernfalls gerade die armen Staaten auf ihren Souveränitätsansprüchen beharren werden (Bull, 1977, S. 288-292)

\section{Institutionalismus}

Eine Gegenposition zum Realismus, die anders als der Idealismus der $Z$ wischenkriegszeit nicht primär normativ orientiert ist, bildet sich in den $40 \mathrm{er} \mathrm{Jahren} \mathrm{mit}$ dem liberalen Institutionalismus hexaus. Hervorgehoben werden die wachsende Bedeutung transnationaler Kooperation beim Versuch der Bewältigung gemeinsa- 
mer Probleme, die steigenden Kosten, die sich für Staaten aus nicht-kooperativem Verhalten ergeben, sowie die Fähigkeit internationaler und regionaler Institutionen, Aufgaben zu übernehmen, denen die Staten nicht mehr gewachsen sind. Vor allem der Prozess der europäischen Integration scheint die institutionalistische Hypothese zu verifizieren. Der Begriff der internationalen Politik ist im Urteil von Karl Kaiser (1969) den Realitäten des 20. Jahrhunderts nicht mehr angemessen, weil sich Politik daxin nicht auf zwischenstaatliche Handlungen beschränkt. Findet zwischen nationalen Gesellschaften Interaktion statt, die nicht auf die Ebene der Regierungsinstitutionen begrenzt bleibt, so lässt sich von transnationaler $G e-$ sellschaft und Politik sprechen. Was auf dieser Ebene geschieht, vermag die sozioökonomischen Strukturen eines nationalen Systems selbst gegen dessen Widerstand zu verändern.

Weltpolitik wird im Urceil liberal-institutionalistischer Autoren nicht ausschließlich von Staaten gestaltet, sondern ebenso von multinationalen Unternehmen und Interessengruppen, von internationalen Organisationen und transnational tätigen Nicht-Regierungsorganisationen. Die pluralistische Transformation der Weltpolitik darf jedoch, wie Robert O. Keohane und Joseph Nye (1977) gezeigt haben, nicht idealisiert werden. Integrationsprozesse beschränken sich oft auf die westlichen Industrienationen; Staaten spielen nach wie vor eine zentrale Rolle und ökonomische Interessen sind häufig national determiniert; Macht und Wohlstand sind unter den Nationen ungleich verteilt und ökonomische Interdependenz ist nicht für alle Staaten im gleichen Maß vorteilhaft. Interdependenz schafft immer Kosten für die betroffenen Akteure, die in ihrer Autonomie eingeschränkt werden; nur von Fall zu Fall kann der Nutzen die Kosten übersteigeñ. ${ }^{3}$

Zentrale realistische Annahmen sind für Keohane unverzichtbar. Internationale Institutionen oder Regime (vgl. Little, 1997), das heißt Netzwerke von Regeln, Normen und Verfahren, die das Verhalten der Akteure regulieren und zur Konfliktlösung beitragen, können in seiner Sicht nur erfolgreich sein, wenn Staaten die Chance sehen, auf diesem Weg ihre Interessen besser zu verfolgen. Die realistische Theorie verkennt jedoch, dass die Politik der Staaten nicht nur durch Machtverteilung bestimmt wird, sondern auch durch Informationsyerteilung, welche ihrerseits zur Verstärkung der Kooperation beitragen kann. Staaten bleiben zwar vorderhand die wichtigsten Akteure der Weltpolitik, als rationale Nutzenmaximierer erkennen sie aber die Vorteile der Kooperation und bestimmen ihre Interessen aufgrund eines modifizierten institutionellen Umfelds neu. Unter Institutionen versteht Keohane ein Ensemble von Regeln und Verhaltensnormen, das menschliches Handeln strukturiert. Die wichtigste institutionelle Praxis auf globaler Ebene is seit dem 17. Jahrhundert die legislative Souveränität der Staaten. Die Analyse globaler institutioneller Veränderungen muss daher prüfen, welche Funktion darin der Souveränität zukommt.

3 Die Einschätzung der Resulrate zwischenstaatlicher Interaktion wird häufig als wichtiger Un terschied zwischen Neorealismus und Neoliberalismus angeführr, vgl. Baldwin, 1993, S. 4-8. Während Neorealisten Wettbewerb und Kooperation als Nullsummenspiel betrachten, sehen Weoliberale darin ein Positivsummenspiel. Keohane und Nye vertreten diesbezüglich eine differenzierte Position: Keohane/Nye, 1977, S. $9 \mathrm{f}$
Keohane bekennt sich zur aufgeklärten Grundüberzeugung, wonach menschliche Praxis institutionelle Realitäten gestalten und verändern kann. Er misstraut aber den Annahmen des republikanischen wie des ökonomischen Liberalismus. Friedfertig sind Republiken vor allem im Umgang mit anderen Republiken, und Freihandel führt nicht notwendig zu Frieden und Harmonie. Kooperation erfordert Planung und Regulierung, der Glaube an die Überlegenheit von Märkten gegenüber staatlicher Wirtschaftsregulation ist mit dem institutionalistischen Ansatz daher inkompatibel. Für Anliegen globaler Gerechtigkeit ist dieser Ansatz offen. Eingeräumt wird, dass internationale ökonomische Regime, die vorwiegend die Interessen hochentwickelter Industriestaten repräsentieren, in moralischer Hinsicht unzulänglich sind und Rawls' Differenzprinzip widersprechen. Das Problem besteht darin, umfassendere Formen der Kooperation zu schaffen, die das Eigeninteresse der Akteure berücksichtigen und dennoch Gerechtigkeitskriterien entsprechen. ${ }^{4}$

Institutionalistische und Regime-Ansätze werden mittlerweile durch das Konzept dér "global governance" bereichert. Mit "governance" wird die Gesamtheit von Ordnungssystemen bezeichnet, die in der Gesellschaft zur Anwendung kommen. "Governance" bedeutet nicht "government". Bestimmte Aufgaben müssen, wie James Rosenau darlegt, in jedem lebensfähigen menschlichen System erfüll werden, unabhängig davon, ob es Institutionen speziell zu diesem Zweck auscebildet hat. Regelsysteme können sich erhalten und ihre Kontrollfunktion effizien ausüben, auch wenn eine etablierte rechtlich-politische Autorität fehlt. Verlieren die Staaten im Prozess beschleunigten Wandels ihre Regierungsfähigkeit, bedeute dies nicht zwingend, dass die entsprechenden Aufgaben nicht mehr erfüllt werden. Während „government" eine als Autorität anerkannte und mit polizeilicher Sarktionsmacht ausgestattete Vollzugsgewalt unterstellt, umfasst "governance" zusätzlich Aktivitäten, die sich nicht von formellen, gesetzlich definierten Verantwortlichkeiten herleiten lassen und die ohne polizeliche Sanktionsmöglichkeiten erfolgreich sein können. Der Begriff "governance without government" ist, wie Rosenau glaubt, geeignet, eine ohne zentrale Autorität funktionierende Weltpolitik zu analysieren (Rosenau, 1992a, S. 3-9; 1998, S. 29 f

\section{Liberaler Internationalismus}

Der liberale Internationalismus hat seine Wurzeln in der Aufklärung und in der liberalen Wirtschaftslehre. Liberale Theorien internationaler Politik (vgl. Zacher/ Matthew, 1995) beruhen auf dem Vertrauen in die Macht der Geschichte, ökonomische, soziale, intellektuelle, moralische Kräfte freizusetzen, die langfristig einen Zustand des Friedens und der Harmonie zwischen Staaten und Menschen herbeizuführen vermögen. Praktiken der Konflikrtverhütung durch Institutionalisierung von Rechtsnormen, die sich innerhalb der Nationalstaaten bewährt haben, sind dieser Auffassung zufolge auch im internationalen Bereich anzuwenden. Im Zeit-

\footnotetext{
4 Keohane, 1984, S. 243-257; 1986, S. 190-200; 1989, S. 7-11, 162-166; 1993.
} 
alter der Französischen Revolution findet sie ihre wichtigsten Vertreter in Denkern wie Immanuel Kant und Jeremy Bentham, der übrigens als Erfinder des Wortes „international“ gilt (Bentham, 1996, S. 296). In seiner 1786 geschriebenen, aber erst in der Mitte des 19. Jahrhunderts veröffentlichten Schift "A Plan for an Universal and Perpetual Peace erkennt Bentham die Chance für eine Verhinderung des Krieges zwischen Staaten in einer internationalen Gesetzgebung und einem internationalen Gerichtshof. Sein Vertrauen setzt er indes primär in die Entfaltung des Freihandels.

Die Überzeugung, das Problem der Errichtung einer innerstaatlichen Rechrsordnung sei nur unter der Bedingung zu lösen, dass auch die Verhältnisse zwischen den Staaten gesetzmäßig geregelt werden, vertritt Kant 1784 in seiner „Idee zu einer allgemeinen Geschichte in weltbürgerlicher Absicht". Die Staaten sollen den gesetzlosen Zustand überwinden und sich in einem Völkerbund zusammenschließen, worin sie als gleichberechtigte und autonome Vetragspartner gelten, als moralische Personen, wie es in der 1795 veröffentlichten Schrift "Zum ewrigen Frieden" heißt. Kant erhebt darin die Forderung, jeder Staat solle republikanisch verfasst sein. Nur die republikanische Verfassung geht aus der Idee des ursprünglichen Vertrags hervor. Obgleich aber der anzustrebende Völkerbund ebenso der Idee einer Überwindung des Naturzustands mittels eines Vertrags entspricht, stellt er keine souveräne Gewalt dar, sondern eine Genossenschaft, deren Aufgabe in der Verhinderung des Krieges zwischen den Staaten besteht. Das Völkerrecht soll mithin auf einen Föderalismus freier Staaten, die ja ihrerseits bereits rechtlich verfasst sind, gegründet sein. Kant räumt freilich ein, "nach der Vernunft" müssten die Staaten einen Völkerstaat, eine Weltrepublik bilden (Kant, AA, Bd. 8, S. 15-31; 341 386; Bd. 6, S. 343-355).

Kants Schrift lässt unterschiedliche Deutungen zu. ${ }^{5}$ Der Warnung vor dem seelenlosen Despotismus einer Weltregierung kann systematisch nicht das gleiche Gewicht zukommen wie der Herleitung der "positiven Idee" einer Weltrepublik. Der Staatenbund ist ein negatives Surrogat, eine Verlegenheitslösung, die keine Garantie gegen den Ausbruch neuer Kriege bietet. Hinsich tlich der Ursachen der kriegerischen Neigungen von Staaten ist Kants Auskunft nicht eindeutig. Er spricht sowohl von der Bösartigkeit der menschlichen Natur als auch von der in monarchischen Staaten praktizierten Arbeitsteilung, die es Herrschern erlaubt, die Untertanen für thre eigenen Interessen zu instrumentalisieren. Die zweite Ursache fallt in republikanisch verfassten Staaten weg, da die Staatsbürger als rationale Nutzenmaximierer einem Krieg angesichts der damit verbundenen Kosten nicht zustimmen. Whe steht es aber mit der ersten Ursache, der nicht durch das politische System, sondern die Natur bedingten Gewalttätigkeit von Individuen und Völkern? Schon hier schafft der Eigennutz Abhilfe: Kriege sind der Entfaltung des Handelsgeistes abträglich. Als Vertragstheoretiker muss Kant konsequenterweise den Weltstaat bejahen und den selbstsüchtigen Neigungen der Menschen miss-

5 Vgl. Höffe, 1995; Merkel/Wittmann, 1996; Lutz-Bachmana/Bohman, 1996. Zu einer kontroversen Erörterung der Frage, ob Kane die Weltrepublik oder den Staatenbund als beste Lösung empfohlen hat: Laberge, 1998; Teson, 1998. trauen; als Geschichtsphilosoph vertraut er dagegen einem Naturmechanismus, der mit der Intensivierung des Handelsgeistes die globalen Verhältnisse befriedet und die Voraussetzungen für einen funktionierenden Staatenbund schafft, ohne das Prinzip nationalstaatlicher Autonomie in Frage zu stellen. Die beiden Thesen von der Kriegsaversion einer bestimmten politischen sowie jener einer bestimmten ökonomischen Ordnung sind kontrovers diskutiert worden. Systematisch ist die erste These sowohl bei Kant selbst wie bei seinen Nachfolgern wichtiger. Die Hypothese einer natürlichen Harmonie aller egoistischen Einzelinteressen weltweit ist durch kolonialistische und imperialistische Praktiken erschüttert worden; Märkte entstehen nicht spontan, sondern setzen staatliche Regulierung voraus, die ihrerseits nicht $\mathrm{zw}$ ingend friedensfördernd ist. ${ }^{6}$

Neuere im Umfeld des liberalen Internationalismus erschienene Arbeiten thematisieren den Zusammenhang von Demokratie und Frieden, die Chancen einer globalen Rechtsordnung und das wünschbare Maß ihrer institutionellen Ausgestaltung. Michael Doyle $(1995 ; 2000)$ schränkt Kants These von der Kriegsaversion republikanischer Staaten auf den Umgang liberaler Staaten untereinander ein. Darunter versteht er politische Ordnungen, worin fundamentale Bürgerrechte garan." tiert sind, die effelktive Souveränität einer gewählten Legislative zukommt, das Recht auf Privateigentum anerkannt ist und ökonomische Entscheidungen den Gesetzen von Angebot und Nachfrage gehorchen. John Rawls $(1996 ; 1999)$ hat ein vertragstheoretisch begründetes liberales Völkerrecht vorgeschlagen, worin die Vertragspartner "Völker" sind, deren Mitglieder Institutionen, eine politische Kultur und ein Rechts- und Gerechrigkeitsverständnis teilen. Liberal-demokratische Gesellschaften einigen sich in Rawls' Modell auf Grundsätze wie Selbstbestimmung, Gleichberechtigung, Selbstverteidigung, Achtung der Menschenrechte und eine Beistandspflicht benachteiligten Völkern gegenüber. Diesem liberalen internationalen Recht können, so das Argument, auch nicht-liberale Völker zustimmen, vorausgesetzt, es handelt sich um ${ }_{n}$ decent peoples" ${ }^{\prime}$. Diese sind friedliebend, und obgleich ihre Politik durch religiöse oder sonstige umfassende Lehren bestimmt wird, achten sie Menschenrechte und kompensieren das Fehlen von Demokratie und Bürgerrechten durch eine die gesellschaftlichen Gruppen einbeziehende Konsultationshierarchie. Ausgeschlossen aus dem liberalen Völkerrecht bleiben gemäß Rawls Staaten, die dessen Prizipien verletzen, sowie Gesellschaften, die aus historischen oder ökonomischen Gründen nicht zu wohlgeordneten Völkern werden könner.

Im Gegensatz zu Rawls nimmt Jürgen Habermas die kosmopolitischen Implikationen yon Kants Friedensschrift ernst und hält daher dessen Begriff eines die einzelstaatliche Souveränität respektierenden Völkerbunds für inkonsistent. Die Völkergemeinschaft müsste ihm zufolge mit einer Legitimität und Sanktionsmacht ausgestattet sein, die es ihr erlaubt, das Weltbürgerrecht notfalls auch gegen Einzelstaaten durchzusetzen und derart jedem Individuum die Mitgliedschaft in einer Assoziation freier und gleicher Weltbürger zu gewähren. Voraussetzung für eine sol-

6 Eine kritische Einschärzung der ökonomischen Dimension des Liberalen Internationalismus finder sich bei Burchill, 1996 . 
che Veränderung wäre die Transformation der Vereinten Nationen in eine über das Gewaltmonopol verfügende, Legislative, Exekutive und Judikative umfassende Institution (Habermas, 1996b, S. 208-219). Habermas' Auseinandersetzung mir dem durch die Globalisierung bewirkten Souveränitätsverlust der Nationalstaaten ist von der Sorge motiviert, in diesem Prozess müssten demokratische Selbstgesetzgebung und Partizipation illusionär werden und die Chancen einer demokratisch legitimierten wirtschafts- und sozialpolitischen Gestaltung schwinden. Ein politischer Rahmen für die deregulierte Weltwirtschaft ist für ihn daher unverzichtbar, mithin auch die Schaffung zwar keines Weltstaats, aber doch größerer politischer Einheiten auf regionaler oder globaler Ebene (Habermas, 1998, S. 91-169).

Die Idee eines Weltstaats verteidigt hingegen, wenn auch in moderater Weise, Otfried Höffe (1998; 1999). Die Weltrepublik ist in seinem Urteil die von der Vernunft gebotene Rechts- und Staatsform globaler menschlicher Koexistenz, sie darf jedoch nur die Gestalt eines föderalen Komplementär- oder Minimalstaats annehmen und das Existenzrecht sowie die Eigenverantwortung von sich auf Menschenrechte und Volkssouveränität verpflichtenden Staaten nicht negieren. Staatliche Aufgaben übernimmt sie mithin nur subsidiär. Sie ist für den internationalen Frieden sowie für das internationale und weltbürgerliche Recht zuständig. Thre Verwirklichung kann als Ergebnis einer langen Übergangsphase ins Auge gefasst werden, wobei am Ende dieses Prozesses die Einrichtung einer globalen Legislative, Exekutive und Judikative stehen muss.

\section{Demokratischer Internationalismus}

Als eigenständige akademische Disziplin hat sich die Theorie der internationalen Beziehungen unmittelbar nach dem ersten Weltkrieg etablierr. Liberale oder idealistische Programme haben mit der von Woodrow Wilson 1918 verkündeten demokratischen Weltrevolution und der Gründung des Völkerbunds 1919 einen weltpolitisch bedeutsamen Niederschlag gefunden. Die Gründung des ersten Lehrstuhls für Internationale Beziehungen 1919 in Aberystwyth war mit der Hoffnung verbunden, diesen Programmen eine wissenschaftliche $B a s i s$ zu verleihen. Ihrem ursprünglichen Selbstverständnis zufolge bestand die Aufgabe der Disziplin vorrangig darin, einen Beitrag zur Verhinderung künfiger Kriege zu leisten Der liberale Anspruch, die Welt zu verändern und zu verbessern, ist von der realistischen Kritik als idealistisch kritisiert worden. Zwar wird heute $z$ wischen liberalem Internationalismus und Idealismus in der Literatur häufig unterschieden, eine klare Trennung ist aber nicht möglich. Als idealistisch werden Auffassungen bezeichnet, die den anzustrebenden Frieden nicht als Ergebnis historischer Prozesse, sondern einer aktiven Gestaltung internationaler demokratischer Institutionen begreifen (Hollis/Smith, 1990, S. 17-20; Dunne, 1997).

Wichtige Faktoren internationaler Politik sind in internationalistischer wie in idealistischer Sicht demokratische Staatsverfassungen, wobei Anhänger der ,neoidealistischen " (Dunne, 1997, S. $156 \mathrm{ff}$ ) Richtung oft von einem radikalen Demokratiekonzept ausgehen. So ist laut Ennst-Otto Czempiel (1996) die entscheidende
Friedensursache in Kants Argumentation die effektive demokratische Mitbestimmung. Diese ist bis heute auch in westlichen Demokratien nicht garantiert, da große Bevölkerungskreise faktisch von außenpolitischen Entscheidungsprozessen ausgeschlossen sind. Unter dem Prozess der Demokratisierung versteht Czempie die Emanzipation der Gesellschaften von ihren polirischen Systemen. Die Gesellschaftswelt wird ihm zufolge auch im internationalen Bereich andere Strategien verfolgen als die Staatenwelt, wobei aber vorderhand nicht zu erwarten ist, dass sie eine friedlichere Welt schaffen wird (Czempiel, 1993, S. 105-132). Der von Alan Gilbert (1999) vertretene demokratische Internationalismus basiert ebenfalls auf der Annahme, Weltpolitik werde zunehmend von nicht-staatlichen Akteuren mitgestaltet. Gilbert glaubt überdies, das sicherheitspolitisch definierte "national interest" habe im demokratisch ermittelien "common good " Konkurrenz erhalten. Formen demokratischer Gegenmacht, insbesondere die internationale Solidarität radikaler Bewegungen stellen ihm zufolge heute ernstzunehmende politische Kräfte dar.

Int Rahmen globaler Demokratisierungshoffnungen und -strategien sind in den $90 \mathrm{er}$ Jahren neue Visionen von "global governance entstanden. Unter "governance" verstehen die Mitglieder der "Commission on Global Governance" (1995, S. 2-7) die Gesamtheit von Praktiken, mit denen Individuen; offentliche und private Institutionen thre gemeinsamen Angelegenheiten regeln. Betont wird dabei, dass die angestrebte "global governance" auf ein weltweites Engagement für Demokratie angewiesen ist. Die Diskussion über Formen und Chancen kosmopolitischer Demokratie hat sich in den letzren Jahren intensiviert (Archibugi/Held, 1995; Archibugi/Held/Köhler 1998).

David Held (1995) verwendet in seiner Untersuchung der Aussichten globaler Demokratisierung einen umfassenden Politikbegriff, der über den Bereich von Staat und Regierung hinaus den gesamten Bereich der Macht bezeichnet. Unter Macht versteht er die Fähigkeit von Individuen und Institutionen, ihre natürliche und soziale Umwelt zu erhalten und zu verwandeln. Legitimitätskriterium einer solchen Politik ist das Autonomieprinzip. Menschen sollen gleiche Rechte und Pflichten haben bei der Gestaltung der politischen Verhältnisse, welche die Spielräume und Grenzen ihres Handelns abstecken. Sie haben Anspruch auf spezifische Rechte für sämtliche Bereiche, in denen Macht ausgeübt wird und ungleich verteilt ist. Da Macht innerhalb nationaler Grenzen nur mehr beschränkt kontrollierbar ist, müssen verfassungsmäßige Strukturen zum Schutz des Autonomieprinzips innerhalb wie $z$ wischen staatlichen Grenzen garantiert sein; das demokratische öffentliche Recht muss kosmopolitisch sein. Der Vollzug eines solchen Rechts setzt die Schaffung neuer Formen kosmopolitischer Demokratie voraus. Held entwirft ein dezentrales Modell, welches einem sich verdichtenden Netzwerk entspricht, einer Gemeinschaft von Staaten und weiteren Assoziationen zum Zweck der Wahrung des globalen demokratischen Rechts.

Radikaler ist der Ansatz von Richard Falk, der sich im Rahmen des, World Order Models Project" seit Jahrzehnten bereits mit Problemen demokratischer Weltordnung und globaler Verfassung befasst. Falk umschreibt die globalen Integrationstendenzen der Gegenwart, die die Handlungsmöglichkeiten souveräner Territorialstaa- 
ten vermindern, mit dem Begriff "geogovernance*. Diese ist bestimmt durch die Interessen der führenden Industriestaaten und die Imperative des kapitalistischen Weltmarkts. Eine die staatliche Einheit fragmentierende Identitätspolitik, globale Apartheid und ein Wohlstandsgefalle auf allen Ebenen, selektiver Menschenrechrsschutz, Unsicherheit, das Fehlen einer wirksamen Umweltpolitik sowie die bescheidenen Aussichten auf eine internationale Demokratisierung sind weitere Merkmale dieser Weltordnung. Als Alternative zum status quo schlägt Falk das Konzept der "humane governance" vor; es umfasst die Realisierung der Menschenrechte inklusive ökonomischer und sozialer Rechte, die Ausdehnung von Partizipationsmechanismen sowie die Schaffung von Verfahren, die es erlauben, globalpolitische und globalökonomische Entscheidungsträger zur Verantwortung zu ziehen. „Humane governance" zielt auf eine globale Wirtschafts- und Sozialpolititik sowie auf die Stärkung der internationalen Gerichtsbarkeit. $\mathrm{Da}$ die Kontrolle der Weltmarktkräfte zu den dringlichsten Aufgaben gehört, hält Falk eine Reform der Weltordnung ohne den Einsatz souveräner, demokratisch legitimierter Staaten für unrealistisch (Falk, 1995 , S. 223-255; 1998a, S. 38-45).

\section{Strukturalismus}

Die dritte Großtheorie führt in der zeitgenössischen Diskussion ein eher randständiges Dasein, was wohl auch politische Gründe hat. Der Strukturalismus hat seine Wurzeln in der Theorie von Marx, der die determinierende Rolle ökonomischer Strukturen sowie die dem Kapitalismus inhärente Tendenz zur Globalisierung analysiert hat. Marxistisch inspirierte Imperialismustheorien sind zu Beginn des 20. Jahrhunderts einflussreich gewesen. In den 60er Jahren stellt der Entwicklungsrückstand ärmerer Länder eine theoretische Herausforderung dar. Mit Problemen ungleicher Entwicklung haben sich damals internationale Organisationen wie ECLA (Economic Commission on Latin America) und UNCTAD (United Nations Conference on Trade and Development) befasst. ${ }^{7}$ Dependenztheoretische Ansätze vertreten zunächst vor allem lateinamerikanische Sozialwissenschafter Unterentwicklung ist das Resultat einer jahrhundertelangen globalen Geschichte; sie erklärt sich nicht primär aus der Existenz vorkapitalistischer Strukturen in den betreffenden Ländern, sondern umgekehrt aus deren Integration in den kapitalistischen Entwicklungsprozess, so lautet die These von André Gunder Frank. Eine eigenständige industrielle Entwicklung ist diesen Ländern verwehrt, sie befinden sich gegenüber den Metropolen der kapitalistischen Weltwirtschaft in einem Satellitenstatus und sind für den Weltmarkt hauptsächlich als Rohstoffexporteure von Interesse (Frank, 2000).

Die Überzeugung, die Analyse der gegenwärtigen Weltwirtschaft setze das Studium ihrer langfristigen Entstehungsgeschichte voraus, liegt auch der von Imma-

7 Zur Entstehung und politischen Bedeutung des Strukturalismus vgl. Blomström/Kettne, 1988 Brown, 1985; Viotti/Kauppi, 1990, S. 406-418; Brown, 1997, S. 186-206; zum Weltsystemansatz Bornschier, 1993 nuel Wallerstein begründeten Weltsystemtheorie zugrunde (Wallerstein, 1974ff.). Wallerstein zufolge hat sich im Zuge der Durchsetzung einer internationalen Arbeitsteilung $z$ wischen dem 16. and dem ausgehenden 19. Jahrhundert ein weltumfassendes kapitalistisches System herausgebildet. Die Welt wurde eingeteilt in zentrale, semi-periphere und periphere Regionen, wobei mächtige Staaten, die in der Arbeitsteilung über günstige Positionen verfügen, das Zentrum bilden. Zentrum und Peripherie unterscheiden sich durch die Höhe des Profits und der Arbeitskosten sowie durch unterschiedliche Formen der Kontrolle über die Arbeit, Formen, die in peripheren Regionen die Gestalt von Sklaverei annehmen können. Der Zweck der Arbeitsteilung ist die Akkumulation von Kapital, das Mittel dazu ein grenzüberschreitender Warenverkehr, der einen permanenten Mehrwert-Transfer von der Peripherie zum Zentrum bewirkt. Die Präferenz der Kapitalisten gillt dabei jenen wirtschaftlichen Aktivitäten, die am ehesten monopolisiert werden können. Weil souveräne Staaten die Monopolbildung erlauben und die Existenz relativ freier Märkte sowie die Kontrolle über die Arbeit garantieren, sind sie ein unverzichtbarer Bestandreil des Systems. Auf globaler Ebene entspricht eine stabile, hegemonial dominierte internationale Ordnung dem Ziel der Kapiralakkumulation; so lauten einige Thesen von Wallerstein (1996)

Als marxistisch kömnen solche Auffassungen nur bedingt gelten. Sie sind denn auch von marxistischer Seite kritisiert worden, die Dependenztheorie wegen eines romantisch-rnationalistischen Antikapitalismus, die Weltsystemtheorie wegen der Fixierung auf die Zirkulationssphäre und der Vernachlässigung der Produktionssphäre. Der marxistische Wirtschaftshistoriker Robert Brenner weist darauf hin, dass die Kapitalakkumulation nicht auf einem Mehrwert-Transfer von der Peripherie ins Zentrum beruht, sondern auf der durch technische Innovation ermöglichten Steigerung der Arbeitsproduktivität (Brenner, 1977; vgl. Sitton, 1996, S. 200-245).

Marxistisch beeinflusst ist auch die Kritische Theorie der Internationalen Beziehungen. ${ }^{8}$ Diese hält, so die Definition von Robert Cox, die bestehende Weltordnung nicht für selbstverständlich, sondern richtet die Aufmerksamkeit auf ihren Ursprung und auf Prozesse des historischen Wandels. Zentraler Gegenstand eines historisch-materialistischen Forschungsprogramms ist der als Machtbeziehung verstandene Produktionsprozess. Handlungsmöglichkeiten sind in dieser Sichtweise weitgehend bestimmt durch die Interaktion von materiellen Fähigkeiten Ideen und Institutionen. Die Analyse der internationalen Beziehungen muss daher die durch den Produktionsprozess erzengten sozialen Kräfte, die Staatsformen und die Weltordnungen umfassen. Die Chancen politischen Handelns sind nie allein von zwischenstaatlichen Machtkonstellationen abhängig, sondern wesentlich von Einflüssen und Zwängen, die von der materiellen Welt ausgehen (Cox, 1986). ${ }^{9}$

Das Interesse an strukturalistischen oder marxistischen Erklärungsmodellen scheint heute zu wachsen. Gründe dafür sind das Bedürfnis, globale Strukturen im

8 Vgl. Linklater, 1990; 1996c. Andrew Linklaters Version der Kritischen Theorie orientiert sich an Habermas' Marx-Kritik und an dessen Diskursethik

9 Vgl. auch Cox, 1987; zur Globalisierung Cox, 1994; zu deren politischen Auswirkung Cox 1997. 
Kontext langfristiger historischer Transformationen zu analysieren (vgl. Buzan/ Little, 2000), sowie Einsichten in die Abhängigkeit der Staaten von der hoch integrierten und schwach regulierten Weltwirtschaft. ${ }^{10}$ Die Erfahrung, dass die ökonomische Globalisierung bisherige Formen politischen Handelns in Frage stellt, ist ein Leitmotiv der neueren Literatur. Dem kapitalistischen Weltmarkt entspricht keine Weltgesellschaft mit regulierenden Institutionen, so konstatieren Elmar Altvater und Birgit Mahnkopf (1999, S. 72-81; 478-516). Globalisierung heißt daher vorderhand nur, dass zahlreiche politisch folgenreiche Entscheidungen nicht mehr von einer demokratisch abgestützten und zu verantwortenden Politik gefällt werden. Die Bedeutung des Marxismus für die Theorie der Internationalen Beziehungen ${ }^{11}$ ist indes schwer einzuschätzen, da eine marxistische Theorie der internationalen Politik, die sich nicht auf die Nord-Süd-Beziehungen beschränkt und gleichzeitig dem radikalliberalen Erbe der Aufklärung verpflichtet ist, ein Desiderat bleibt (Halliday, 1994, S. 47-73).

\section{Reflexive und konstruktivistische Ansätze}

In jüngerer Zeit sind realistische, liberale und strukturalistische Theorien wegen ihrer angeblich positivistischen Epistemologie unter Beschuss geraten. Fragen nach Standpunkt und Interesse der Forschenden oder nach dem Einfluss selektiver Wahrnehrnung auf das Handeln wird stärkeres Gewicht beigemessen. Realistische Theorien, so wird häufig vermutet, tragen dazu bei, jêne Wirklichkeit, auf die sie sich beziehen, überhaupt erst zu konstruieren. Folgt die Regierung einer Großmacht den Lektionen der realistischen Schule, wird ihre Politik wahrscheinlich das realistische Erklärungsmodell bestätigen. Die Erkenntnis, dass die Welt nicht auBerhalb unserer Theorien über sie existiert, sondern dass Theorien uns helfen, eine Welt zu konstruieren, worin wir uns zurechtfinden und agieren können, mithin bis zu einem gewissen Grad immer auch „self-fulfilling prophecies" sind, hat zu einem Aufstand gegen die Vorherrschaft der "positivistischen" Schulen, insbesondere des Realismus mit seinem Objektivitätsanspruch geführt (vgl. Smith, 1996; 1997).

In einem frühen Stadium der Auseinandersetzung hat Keohane eingeräumt, die Einwände der von ihm so benannten reflexiven Denkschule seien teilweise berechtigt (Keohane, 1989, S. 158-179). Rationalistische Ansätze gehen oft von unhistorischen Voraussetzungen aus und verdrängen, dass die Präferenzen der Akteure nicht einfach gegeben sind, sondern durch historische Entwicklungen, institutionelle und kommunikative Praktiken sowie soziale Lemprozesse geformt werden. Keohane verlangt von rationalistischen Modellen, dass sie die Ausgangsbedingungen berücksichtigen, unter denen Akteure sich entscheiden müssen. Die reflexive Kritik bleibt in seinem Urteil wissenschaftlich jedoch folgenlos, da ihr ein kohärentes Forschungsprogramm fehlt.

10 Vgl. dazu die Arbeiten von Susan Strange, 1988; 1995; 1996; Stubbs/Underhill, 1994; Tooze,

11 Ausführliche Diskussionen finden sich bei Linklater, 1990; Halliday, 1994; vgl. auch Maclean, 1988; für einen knappen Uberblick Smith, 1994; Linklater 1996b.
Den Versuch, den Graben zwischen rationalistischen und reflexiven Ansätzen zu überbrücken, hat einige Jahre später Alexander Wendt unternommen. Der neoliberale Institutionalismus befindet sich ihm zufolge in einem Dilemma. Sein wissenschaftliches Ziel besteht darin, Lernprozesse zu erklären, die kooperatives Verhalten bewirken. Die ökonomische Theorie der rationalen Entscheidung ist ihm dabei nicht dienlich; angewiesen wäre er auf soziologische und psychologische Theorien der Bildung und Veränderung von Identitäten, Interessen und Präferenzen im Prozess der Interaktion. Wendt sieht das Verdienst der von ihm als konstruktivistisch bezeichneten Ansätze darin, dass sie über solche Theorien verfügen. Die neorealistische Auffassung, wonach die internationale Anarchie gleichsam naturgegeben ist, hält er für falsch; die Anarchie ist ein soziales Konstrukt, ein Produkt institutioneller Praktiken; , anarchy is what states make of it" (Wendt, 2000, S. 617).

Eine grundsäzzliche Rationalismuskritik, wie sie in postmodernen oder poststrukturalistischen Ansätzen gefordert wird, dürfte der Forschung kaum dienlich sein (vgl. Halliday, 1996). Es bleibt, wie Martin Hollis (1996) gezeigt hat, unklar, was unter Post-Positivismus zu verstehen ist und ob damit das Projekr der Aufklärung insgesamt verabschiedet werden soll. Eine aufgeklärte, in der Tradition von Marx und Weber stehende Sozialwissenschaft glaubt an die Möglichkeir objektiver Erkenntnis, richter ihre Aufmerksamkeit aber zugleich auf das die objektive Realität gestaltende und verändernde subjektive Handelin. Die Existenz von Institutionen ist für sie freilich dann unstrittig, wenn diese sich auf menschliche Handlungsmöglichkeiten auswirken, wobei zunächst unerheblich ist, ob sie naturgegeben oder konstruiert sind.

\section{Deskriptive und normative Theorie}

Als eigenständiger Beitrag zur Disziplin der Internationalen Beziehungen gelten in neueren Lehrbüchern normative Theorien. ${ }^{12}$ Das Spannungsverhältnis zwischen Souveränität und Menschenrechten, das Selbstbestimmungsrecht der Völker, die Rechtmässigkeit von Interventionen in die inneren Angelegenheiten von Staaten oder die Verteilung von Nutzen und Lasten im globalen Kooperationssystem sind Forschungsthemen solcher Theorien. Allerdings können empirische Theorien internationaler Politik auf normative Annahmen nicht verzichten, und normative Theorien stützen sich notwendig auf empirisch überprüfbare Annahmen über menschliche Fähigkeiten und Bedürfnisse oder über die Macht und Wandlungsfähigkeit von Institutionen. Dennoch ist es sinnvoll, zwischen deskriptiver und normativer Theorie zu unterscheiden. Empirische Theorien, die den Zustand der Welt beschreiben, haben wissenschaftlichen Kriterien zu genügen. Normative Theorien können dagegen nicht verifiziert oder falsifiziert werden; ob etwas der Fall sein soll oder nicht, ob eine bestimmte Form der Machtausübung legitim ist oder nicht,

12 Vgl. dazu Beitz, 1979 (ein frühes Werk der kosmopolitischen Richtung); Beitz/Cohen/Scaxlon/Simmons, 1985; Ellis, 1986; Brown, 1992; Nardin/Mapel, 1992; Thompson, 1992; Forbes/ ristische Richrung); Mapel/Nardin, 1998; Cochran, 1999. 
ist nicht empirisch überprüfbar. Der Wahrheitsgehalt normativer Theorien hängt von ihrem Erfolg in vernünftigen Diskursen ab. Es ist aber vielleicht möglich, zu überprüfen, ob politische Ordnungen, die als legitim anerkannt werden, langtristig eine größere Stabilität aufweisen als solche, die nicht als legitim anerkannt werden. Selbstrerständlich sind solche Überprüfungen heikel, wie exemplarisch Rawls Wortwahl zeigt, wenn er von "stabilizy for the right reasons" spricht (Rawls, 1999 12f.). Empirisch lässt sich überprüfen, ob eine Ordnung stabil ist und mehrheitlich anerkannt wird, taum überprüfen lässt sich hingegen, ob die tiefsten Motive, die die Akteure eine Ordnung unterstützen lassen, „richtige Gründe“ sind. So können Sozialordnungen, die auf einer ungleichen Verteilung von Fandlungsmöglichkeiten und Information beruhen, wegen der beschränkten Urteils-, Handlungs- oder Widerstands săhigkeit der Mehrheit relativ stabil sein, vom Standpunkt moderner Gerechtigkeitstheorien aber dennoch nicht als legitim gelten. Gleichwohl ist die Hoffnung, es möge gelingen, den Abgrund zwischen empirischer und normativer Fragestellung zu überbrücken, ohne einem naturalistischen Fehlschluss zu erliegen, nicht ganz vergeblich.

Ob jemand einen Vertrag aus Eigeninteresse oder aus Gerechtigkeitssinn einhält lässt sich nicht überprüfen, ob der Vertrag eingehalten wird, jedoch schon. Es ist denkbar, dass im Zuge einer Arbeitsteilung normative Theorien Hypothesen über die stabilisierende Wirkung von Prinzipien fomulieren und empirische Theorien Verifikations- oder Falsifikationskriterien definieren. Zweck der Politik ist im Urteil der modernen politischen Philosophie die Herstellung des Friedens als eines Zustands gesellschaftlicher Stabilität, worin alle Menschen über die gleiche Handlungsfreiheit verfügen. Ein Recht auf stabile Verhältnisse gehört mithin zum modernen Verständnis von Gerechtigkeit. Bedingung einer stabilen Ordnung ist die gleichberechtigte Teilnahme aller Betroffenen an politischen Entscheidungen. Bei den Entscheidungsberechtigten wird zwar eine gewisse Kompetenz vorausgesetzt, doch wird angenommen, dass diese Kompetenz hauptsächlich in der Ausübung der politischen Praxis selbst gewonnen wird; breite Partizipation ist daher im Inteder polis resse der Erhaltung einer Gesellschaft die Kontrolle über ihre Lebensbedingungen entzogen wird oder wenn das politische Gemeinwesen insgesamt die für es vitalen Belange nich mhr hontliert ${ }^{13}$ Stabilität im Sinne symmetrischer Reziprozität zwischen nutonomen Menschen lässt sich als Norm begründen. Stabilität als dauerhafte und autonomen Menschen lasst sich als Norm begrunden. Stabilitat abls dau beide Konzeptionen von Stabilität nicht gleichgesetzt werden dürfen, ist kaum zu bestreiten, zeption zieht, von normativen Motiven ausgeht, und dass die normative Reflexion Gerechsozialer Ordnung begreift.

Seit einiger Zeit artikuliert sich in der Theorie der internationalen Beziehungen $^{14}$ das Bedürfnis, über einen den veränderten globalen Realitäten angemessenen

13 Ich fasse Einsichten von Rousseau, Kant, Mill und Marx zusammer.

14 Rosenzu, 1989; 1990, S. 6ff; Brown, 1996, S. 1-12; Williams, 1996, S. 141-160.
Begriff der Politik zu verfügen. Die Differenz zwischen dem "eigentlich“, das heilt innerhalb nationaler Grenzen Iokalisierbaren Politischen und den internationalen Angelegenheiten, $z$ wischen einem gesetzlich geregelten Innenbereich und einem anarchischen Außenbereich ist von Robert Walker (1993) als metaphysisches Konstrukt entlarvt worden. Der auf Vorstellungen von Inklusion und Exklusion aufbauende Politik-Begriff ist fragwürdig geworden, damit aber auch der herkömmliche Begriff internationaler Politik. Einer Situation, in der Staten auch auf globaler Ebene nicht mehr die einzigen Akteure sind, ist er nicht angemessen; er solite, so James Rosenaus Vorschlag, durch einen Begriff post-internationaler Politik ersetzt werden. Die moderne politische Philosophie basiert auf der Überzeugung, jeder Zustand, in dem Menschen sich gegenseitig Schaden zufügen oder sich in ihrer Freiheit einschränken, sei mittels der Errichtung politischer Institutionen überwindbar. Eine Beschränkung der politischen Aufgaben auf einen nationalen Innenbereich ist folglich unzulässig. Das heißt nicht, nur ein Weltstaat vermöge die elementaren politischen Aufgaben zu lösen. Es heißt, dass die moderne Politikn Konzeption beim Entwrux von Ordnungsmodellen nach Möglichkeit von den allen Menschen beziehungsweise allen potentiellen Gegnern im Naturzustand gemeinsamen Motiven ausgehen muss.

\section{Abschließende Bemerkungen}

Dass grundlegende politische Aufgaben künftig nur noch global bewältigt werden können, wenn sich gesellschaftlich-wirtschaftliche Kooperationssysteme globalisieren, versteht sich von selbst. Vom Grad der Integration in ein Kooperationssystem sowie der Betelligung am Nutzen dieser Kooperation hängt es aber ab, inwiefern sich Individuen und Gruppen als Kooperierende mit Rechten und Verantwortungen verstehen. Theorien der internationalen oder globalen Politik enthalten Annahmen über die Rationalität individueller und institutioneller Akteure sowie über die Ausdehnung von Kooperationssystemen. Die realistische Verengung der Sichtweise auf staatliche Politik, die Auffassung, wonach Staaten homogene Gebilde mit einheitlichen Interessen sowie die einzigen Akteure der globalen Politik sind, widerspricht heutigen Realitäten. Das heißt nicht, die Aufmerksamkeit für interessegeleitetes Verhalten und Strategien der Machtakkumulation erübrige sich, es heißt, dass dabei nicht allein die Staaten in Betracht kommen dürfen. Liberale und radikale Ansätze haben gegenüber realistischen den Vorzug, dass sie über ein umfassenderes. Modell potentieller Akteure vertügen und darüber hinaus gewisse normative Prinzipien der Moderne als mögliche Motive politischen Handelns in Betracht ziehen. Sie setzen voraus, dass Menschen rationale und vernünftige Wesen sind, die ein Wissen um die langfristigen Bedingungen erfolgreicher Interessenverfolgung erwerben und danach handeln, aber auch bereit sind, ihren eigenen Freiheitsanspruch mit dem Freiheitsanspruch aller anderen Menschen in Beziehung zu setzen. Gerade liberale Theorien blenden jedoch gerne aus, dass individuelle und kollektive Akteure nur in einem beschränkten Ausmaß frei sind, die Verhältnisse zu gestalten und zu vetändern, weil ihre Handlungsfähigkeit durch strukturelle 
Zwänge machtpolitischer und ökonomischer Art eingeschränkt wird. Strukturalistische Erklärungsmodelle korrigieren den naiven Optimismus liberaler Wirtschaftsdoktrinen; sie orientieren sich an einem nicht harmonischen, sondern antagonistischen Bild sozio-ökonomischer Modernisierung und fragen nach deren Auswirkungen auf das System der Verteilung von Macht und Chancen. Niemand bestreitet, dass eine langfristige Stabilisierung der globalen Verhältnisse bei allen Betroffenen die Bereitschaft voraussetzt, gleiche Rechtsansprüche zu respektieren. Diese Bereitschaft kann aber nur auf der Überzeugung beruhen, im System der Verteilung von Macht, Freiheit und Wohlstand angemessen berücksichtigt zu werden.

Stabilisierung setzt mithin Demokratisierung voraus, wobei unter Demokratie eine Ordnung zu verstehen ist, die gleiche Rechte garaniert, gleiche Chancen, diese Rechte zu nutzen, sowie gleiche Teilnahmemöglichkeiten an politischen Ent scheidungsprozessen. Demokratien führen untereinander keinen Krieg, so lautet ein bekanntlich leicht zu falsifizierendes liberales Theorem. Die Theorie ist folglich auf Hilfshypothesen angewiesen, die es erlauben, zwischen echter und unechter Demokratie zu unterscheiden. Echte Demokratien, die den oben definierten Kriterien entsprechen, würden, so ließe sich das Theorem umformulieren, aus der Welt einen friedlicheren Ort machen, im Wissen darum, dass Verteilungsungerechtigkeit kriegsfördernd sein kann, eine weniger diskriminierende Chancenver teilung anstreben und derart einer globalen Demokratisienung zuarbeiten. Ist diese Hoffnung realistisch? Zweifellos spielen radikaldemokratische Bewegungen heute weltweit eine stärkere Rolle und können sich von Fall zu Fall zu Kräften entwickeln, die den mächtigen staatlichen und wirtschaftlichen Akreuren globaler Politik Widerstand leisten können (Gilbert 1999). Allerdings ist gegenwärtig schwer absehbax, ob und wie diese Bewegungen über Grenzen hinweg kommunizieren, ihre Bemühungen koordinieren und verhindern können, dass demokratische Impulse im nationalen Rahmen von anti-etatistischen, national-populistischen oder religiös-traditionalistischen Kräften instrumentalisiert und für die Verteidigung von Privilegien mobilisiert werden.

Nur eine demokratische Weltordnung, die die Bevölkerungen aller, einschließ. lich armer Länder einbezieht, könnte die bestehenden Macht- und Wohlstandsdisparitäten abbauen; doch es sind gerade diese Disparitäten, die das kosmopolitische Projekt als utopisch erscheinen lassen. Jede Theorie internationaler oder globaler Politik ist mit diesem Paradox konfrontiert und muss sich daher mit Macht befassen. Macht lässt sich nicht auf Staatsmacht reduzieren, wie dies klassisch-realistische Lehren tun. Ein kritischer Realismus hätte Machtbeziehungen auf mehreren Ebenen zu lokalisieren und zu analysieren: auf der Ebene der Staaten wie auf jener der Gesellschaft und der Wirtschaft (vgl. Falk, 1997; Harrod, 1997). Ökonomische Macht wirkt im Bereich von Arbeit und Produktion, in Marktmechanismen, aber auch im rechtlich-politischen Bereich, wie der wachsende Einfluss von Unternehmen auf Regienung und Gesetzgebung zeigt. Wie die wenigen Stichworte zeigen, müssen Theorien der entgrenzten Politik fachliche Grenzen überwinden, um prïfen zu können, welche Kräfte auf die globalen Konstellationen einwirken, sich gegenseitig verstärken oder neutralisieren. Das Bedürfnis nach verbindlichen Regeln in einer unübersichtlichen Welt gehört ebenso dazu wie die Versuchung hegemoni- aler Mächte, die Regeln nach Maßgabe partikularer Interessen zu interpretieren. Gerechtigkeitsvorstellungen, die von allen Menschen geteilt werden, können die globale Politik genauso gestalten wie Kommunikations geteil werden, konnen die essengegensätze zwischen nung. Einsichten in die Bedingungen stabiler Verhältniße, die zur Bereitschaft führen, Macht und Ressourcen gerechter zu vertellen, stehen Illusionen uber Möt lichkeiten, privilegierte Positionen lanofristig halten, stehen lllusionen über Mögund für alle Seiten nachteilige Konflikte $z$ wischen reichen und armen Weltregionen zu provozieren, gegenüber. Die Mechanismen eines deregulierten Kapitalismus wirken ebenso wie politische Projekte und soziale Bewegungen, die eine stärkere Regulierung oder Demokratisierung der Wirtschaft anstreben. Die Schwierigkeit, etwas über das Resultat des Zusammenwirkens und Zusammenprallens dieser Kräfre auszusagen, gibt einen Eindruck von der Aufgabe, die sich einer Theorie der
Weltpolitik heute stellt. 